\title{
A Fully Autonomous Indoor Quadrotor
}

\author{
Slawomir Grzonka Giorgio Grisetti Wolfram Burgard
}

\begin{abstract}
Recently there has been an increased interest in the development of autonomous flying vehicles. Whereas most of the proposed approaches are suitable for outdoor operation, only a few techniques have been designed for indoor environments, where the systems cannot rely on GPS and therefore have to use their exteroceptive sensors for navigation. In this paper we present a general navigation system which enables a smallsized quadrotor system to autonomously operate in indoor environments. To achieve this, we systematically extend and adapt techniques which have been successfully applied on ground robots. We describe all algorithms and present a broad set of experiments illustrating that they enable a quadrotor robot to reliably and autonomously navigate in indoor environments.
\end{abstract}

Index Terms-UAV, Quadrotor, SLAM, Navigation

$\mathbf{I}$ $\mathrm{N}$ recent years, the robotics community has shown an increasing interest in autonomous aerial vehicles, especially quadrotors. Low-cost and small-size flying platforms are becoming broadly available and some of these platforms are able to lift relatively high payloads and provide an increasingly broad set of basic functionalities. This directly raises the question of how to equip them with autonomous navigation abilities. Whereas most of the proposed approaches for autonomous flying [14], [32] focus on systems for outdoor operation, vehicles that can autonomously operate in indoor environments are envisioned to be useful for a variety of applications including surveillance and search and rescue [10]. In such settings and compared to ground vehicles, the main advantage of flying devices is their increased mobility.

As for ground vehicles, the main task for an autonomous flying robot consists in reaching a desired location in an unsupervised manner, i.e., without human interaction. In the literature, this task is known as navigation or guidance. To address the general task of navigation one is required to tackle a set of problems ranging from state estimation to trajectory planning. Several effective systems for indoor and outdoor navigation of ground vehicles are nowadays available [1], [2].

Whereas the general principles of the navigation algorithms, which have been successfully applied on ground robots, could in principle be transferred to flying vehicles, this transfer is not straightforward for several reasons. Ground robots are inherently stable, in the sense that by issuing a zero velocity command results in the robot to smoothly decelerate until it stops. The same does not apply for flying robots that need to be actively stabilized even when they are already in the desired location. Furthermore, due to the fast dynamics of a flying vehicle compared to a ground one all the quantities necessary to stabilize the vehicle should be computed within a short time and with an adequate level of accuracy. Thus, porting navigation systems for ground robots to aerial vehicles requires to fulfill more stringent constraints on both accuracy and efficiency.

In this work, we present the enabling technology for

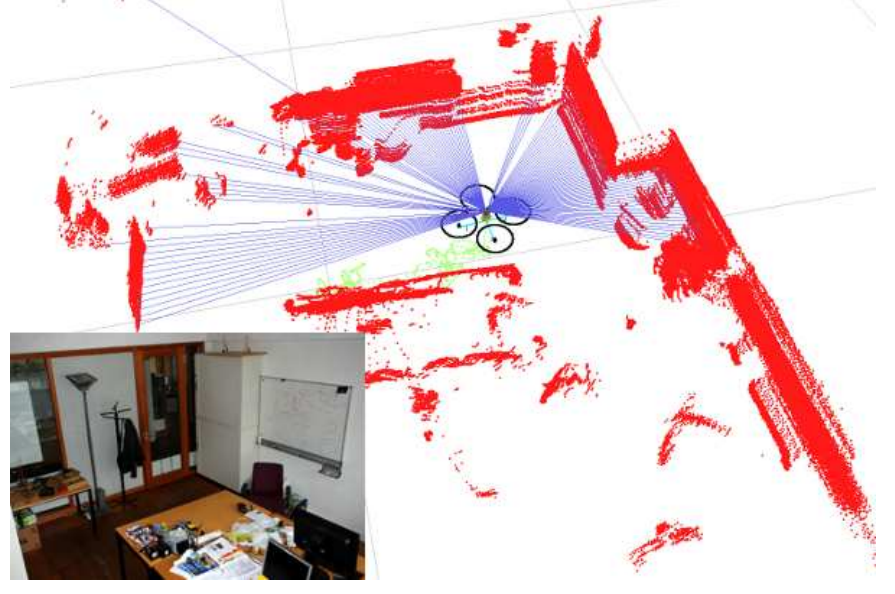

Fig. 1. Autonomous flight of our quadrotor in a cluttered office room. The free space around the robot is seriously confined, imposing high demands on pose stability, localization, and control. The image in the (bottom left) shows the office room from a similar view point as the snapshot.

autonomous quadrotor navigation in indoor environments and describe a navigation system including key functionalities namely localization, planning, surface estimation, maplearning, and control. Whereas a flying vehicle moves in $3 \mathrm{D}$, indoors there is usually enough structure to describe the environment with 2D representations. Instead of using a full $3 \mathrm{D}$ representation we rely on a $2 \mathrm{D}$ one for the walls augmented with the elevation of the floor. The advantage of this choice compared to the full 3D representation is that we can operate in a large class of indoor environments by using efficient variants of 2D algorithms that work on dense grid maps instead of space and time consuming 3D methods. Having these functionalities adapted for the 3D case would be either too slow or not accurate enough given the limited time constraints to make the system stable. This paper extends our previous work [17] by introducing improved algorithms for simultaneously estimating the altitude of the vehicle and the elevation of the underlying surface. We furthermore provide quantitative results of our SLAM approach and discuss the effect of different modes of the incremental scan-matching on the pose stability of the robot. We also describe our algorithms for path planning, obstacle avoidance and provide additional details and experiments.

Our system is a result of an integrated hardware/software design which meets several of the challenging constraints imposed by small size flying vehicles while preserving a large degree of flexibility. It further can be operated at different levels of autonomy. It can be used to assist a pilot by preventing collisions with obstacles and keeping the position of the vehicle when no commands are given. It can construct a map on-line while flying in an unknown environment, or it can be instructed to autonomously reach given locations in a known 
map. We evaluated our system on an open source quadrotor, the so-called the Mikrokopter [3]. Figure 1 visualizes our quadrotor system and its internal state while autonomously flying within an highly cluttered office room.

\section{RELATED WORK}

In the last decade, flying platforms received an increasing attention from the research community. Many authors focused on the modeling and on the control of these vehicles [8], [11], [25], [29], with a particular emphasis on small or micro helicopters [10]. Hoffmann et al. [19] presented a modelbased algorithm for autonomous flying with their STARMACquadrotor. Their system flies outdoors and utilizes GPS and IMU measurements. Ng and colleagues [14] have developed algorithms for learning controllers for autonomous helicopter navigation. Their approach allows helicopters to perform impressive maneuvers in outdoor environments. Scherer et al. [28] describe algorithms for flying fast among obstacles at low altitude using a laser scanner. Tempelton et al. [30] demonstrate how to use vision for outdoor terrain mapping and autonomous landing. Tournier et al. [33] and Bourquardez et al. [12] used vision to estimate and stabilize the current pose of a quadrotor. Thrun et al. [32] used a remotely controlled helicopter to learn large-scale outdoor 3D models. There also has been some work that addressed the navigation of flying vehicles in indoor environments and in absence of the GPS signal. Several authors used vision to control or assist the control of an indoor quadrotor [7], [20], [21]. Roberts et al. [26] used ultrasound sensors for controlling a flying vehicle in a structured testing environment, while $\mathrm{He}$ et al. [18] presented a system for navigating a small-size quadrotor without GPS. Here, the pose of the vehicle is estimated by an unscented Kalman filter. Whenever the robot has to reach a given location, a path which ensures a good observation density is computed from a predefined map. These highly dense observations minimize the risk of localization failures. In parallel to our work, Achtelika et al. [6] developed an indoor autonomous quadrotor equipped with a laser range scanner and cameras enabling autonomous hovering in a constraint indoor environment. Recently, Celik et al. [13] presented their system for indoor simlutaneous localization and mapping (SLAM) using a monocular camera and ultrasound. Our work is orthogonal to a recent work of Bachrach et al, [9] where the authors present a system for performing autonomous exploration and map acquisition in indoor environments. They extend the 2D robot navigation toolkit CARMEN [27] by adding a RaoBlackwellized particle filter for SLAM and an algorithm for frontier-based autonomous exploration. However, they do not provide localization, map optimization, obstacle avoidance or mutli-level SLAM. Furthermore, we utilize a more robust graph-based SLAM algorithm in our system allowing for map optimization and present our algorithm for estimating the altitude of the surface underlying the robot. This enables a quadrotor equipped with our system to fly over surfaces whose height is piecewise constant.

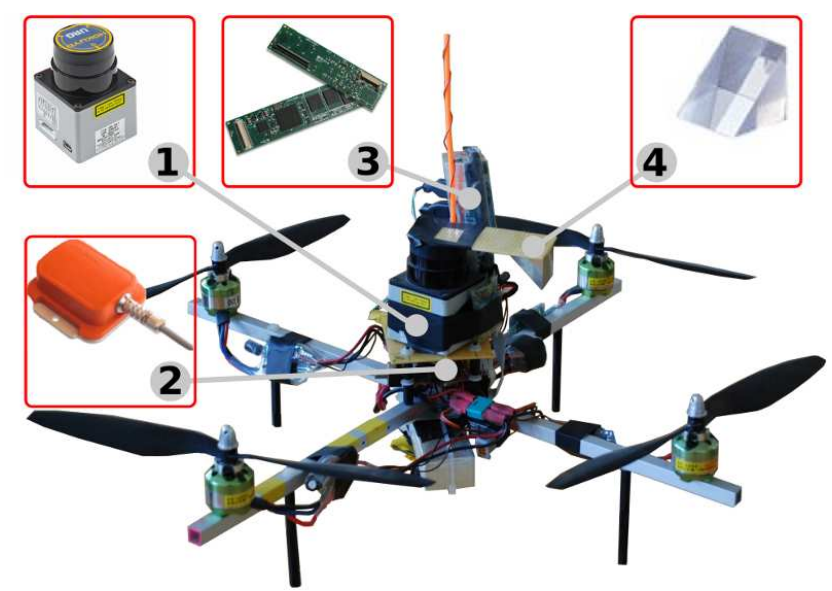

Fig. 2. The quadrotor platform used to evaluate the navigation system is based on a Mikrokopter and includes a Hokuyo laser range finder (1), an XSens IMU (2), a Gumstix computer (3), and a laser mirror (4).

\section{INDOOR NAVIGATION OF AN AUTONOMOUS FLYING QUADROTOR}

To autonomously reach a desired location, a mobile robot has to be able to determine a collision-free path connecting the starting and the goal locations. This task is known as path planning and requires a map of the environment to be known. Usually, this map has to be acquired by the robot itself by processing the sensor measurements obtained during an exploration mission. This task of generating the map is known as simultaneous localization and mapping (SLAM). For most of the applications it is sufficient to perform SLAM off-line on a recorded sequence of measurements. To follow the path with a sufficient accuracy, the robot needs to be aware of its position in the environment at any point in time. This task is known as localization. A further fundamental component of a navigation system is the control module which aims to move the vehicle along the trajectory, given the pose estimated by the localization.

Due to the increased risk of damaging the flying platform during testing, the user should have the possibility of taking over the control of the platform at any point in time. Finally, the more complex dynamics of a flying platform poses substantially higher requirements on the accuracy of the state estimation process than for typical ground-based vehicles. Although in outdoors scenarios, positioning errors up to $1 \mathrm{~m}$ might be acceptable, they are not indoors, as the free-space around the robot is substantially more confined.

\section{HARDWARE ARCHITECTURE}

Figure 2 shows a Mikrokopter [3] open source quadrotor equipped with sensors and computational devices. The Mikrokopter comes with a low level controller for roll, pitch, and yaw. Our quadrotor is similar to the one proposed by He et al. [18] and consists of the following components: an Hokuyo-URG miniature laser sensor for SLAM and obstacle avoidance (1), an XSens MTi-G MEMS inertial measurement unit (IMU) for estimating the attitude of the vehicle (2), a Linux-based Gumstix embedded PC with USB interfaces and a WiFi network card which communicates with the microcontroller on the quadrotor via an RS-232 interface (3), and a 
mirror which is used to deflect some of the laser beams along the $z$ direction to measure the distance to the ground (4).

\section{NAVigation System}

Our navigation system is based on a modular architecture in which different modules communicate via the network using a publish-subscribe mechanism. In our current system all device drivers are executed on-board while the more computationally intensive algorithms run on a remote PC communicating over wireless with the platform.

Since roll $(\phi)$ and pitch $(\theta)$ measured by the IMU are in general accurate up to $1^{\circ}$, we can directly use this information within our navigation system. This allows us to reduce the localization problem from $6 \mathrm{DOF}$ namely $(x, y, z, \phi, \theta, \psi)$ to $4 \mathrm{DOF}$, consisting of the $3 \mathrm{D}$ position $(x, y, z)$ and the yaw angle $\psi$. The only sensor used to estimate these 4DOF and detecting obstacles is the laser range scanner.

Based on known initial calibration parameters and on the current attitude $(\phi, \theta)$ estimated by the IMU, we project the endpoints of the laser into the global coordinate frame. Given the projected laser beams, we estimate the $(x, y, z, \psi)$ of the vehicle in a 2D map containing multiple levels per cell. To compensate for the lack of odometry measurements we estimate the incremental movements in $(x, y, \psi)$ by 2D laser scan-matching. Finally, we control the altitude of the vehicle and simultaneously estimate the elevation of the underlying surface by fusing the IMU accelerometers and the distance from the ground measured by the laser. Accordingly, we track and map multiple levels within an environment, which enables our robot to correctly maintain its height even when flying over obstacles like tables or chairs.

\section{A. Incremental Motion Estimation}

The laser range scanner measures at time $t$ a set of distances $\mathbf{r}_{t}$ along the $x-y$ plane in its own reference frame. We therefore first calculate a projection of the measured distances $\mathbf{b}_{t}$ for the beams not deflected by the mirror using the roll and pitch estimate from the IMU. Consequently, we calculate the points $\mathbf{h}_{t}$ for all beams deflected by the mirror using a chain of transformations from the IMU to the virtual laser position which accounts for the effect of the mirror. Some tasks, like pose stabilization, rely on an accurate local pose estimate of the vehicle in its surroundings. To this end, we can estimate the relative movement of the robot between two subsequent scans by using a scan-matching algorithm. Since the attitude is known from the IMU, this procedure can be carried out in $2 \mathrm{D}$, assuming structured indoor environments. A scan-matching algorithm estimates the most likely pose of the vehicle $\hat{\mathbf{x}}_{t}$ at time $t$ given the previous $k$ poses $\mathbf{x}_{t-k: t-1}$ and the corresponding laser measurements $\mathbf{b}_{t-k: t}$, as follows

$$
\hat{\mathbf{x}}_{t}=\underset{\mathbf{x}:=(x, y, \psi)}{\operatorname{argmax}} p\left(\mathbf{x}_{t} \mid \mathbf{x}_{t-k: t-1}, \mathbf{b}_{t-k: t}\right) .
$$

To solve Equation (1), we use a variant of the multi-resolution correlative scan matcher proposed by Olson [24]. The idea behind a correlative scan-matcher is to discretize the search space $\mathbf{x}_{t}=\left(x_{t}, y_{t}, \psi_{t}\right)$ and to perform an exhaustive search in these discretized parameters around a given initial guess. To efficiently evaluate the likelihood $p\left(\mathbf{x}_{t} \mid \mathbf{x}_{t-k: t-1}, \mathbf{b}_{t-k: t}\right)$ of a given solution $\mathbf{x}_{t}$, we use likelihood fields [31] obtained by the most likely map generated from the last observations $\mathbf{b}_{t-k: t-1}$.

The complexity of a correlative scan-matcher depends linearly on the resolution at which the parameters are discretized and on the search range. A naive implementation of this algorithm is not adequate for our application that demands both high accuracy and efficient computation. To overcome this problem, we employ a multi-resolution approach. The idea is to perform the search at different resolutions, from coarse to fine. The solutions found at a coarse level are then used to restrict the search at a higher resolution.

In our implementation we use a constant velocity model to compute the initial guess for the search and we perform the correlative scan matching at three different resolutions (i.e., $4 \mathrm{~cm} \times 4 \mathrm{~cm} \times 0.4^{\circ}, 2 \mathrm{~cm} \times 2 \mathrm{~cm} \times 0.2^{\circ}$, and $\left.1 \mathrm{~cm} \times 1 \mathrm{~cm} \times 0.1^{\circ}\right)$. We set the search area $r$ depending on the maximum speed $v_{\max }$ of the vehicle and on the frequency $f$ of the scanner as $r=v_{\max } / f$.

We control the position of the vehicle based on the velocities estimated by the scan-matcher. Accordingly, the performances of the scan-matcher play a major role in the stability of the robot. In particular, we want to have a fast, accurate but still smooth (i.e., less oscillations) estimate. To get an intuition about the desired accuracy, consider an error in the position estimate of $\pm 2 \mathrm{~cm}$. Assuming a sensor frequency of $10 \mathrm{~Hz}$ this error leads to a variation of $20 \frac{\mathrm{cm}}{\mathrm{s}}$ in the velocity estimate between two laser scans. This in turn can generate wrong commands by the controller reducing stability.

In our hierarchical scan-matcher, the high-resolution estimate is affected by frequent oscillations due to the limited resolution of the likelihood field. Although these oscillations could in general be filtered out by a low-pass filter, this type of filtering would introduce a phase shift in the pose and velocity estimate (the estimated pose is past in time). To obtain both, an accurate position estimate and a smooth signal, we compute the final solution as the weighted mean of the estimates of all scan-matchers in the hierarchy. The weights of the sum lie on a Gaussian centered at the finest resolution estimate. In several experiments we found that the weighted average of the estimates is better for control as each single estimate as shown in Table I. The table contains experimental results comparing the effect on the pose stability using the estimate of the individual scan-matchers versus our weighted mean approach. All runs reflect experiments where the goal of the quadrotor was to hover at the same spot at $0.5 \mathrm{~m}$ height for as long as the battery holds. To quantitatively evaluate our approach, we compare the mean and standard deviation in both, position and absolute velocity. As can be seen, using a weighted average of the different resolutions has a positive affect on the control loop. This originates from the fact that the weighted averaging has a smoothing effect on the pose estimate but does not include any phase shift into the system. Since we use a simplistic model of our quadrotor (constant velocity model), using the output of the weighted mean (with the prediction used as the initial guess for the search) is 


\begin{tabular}{|l|c|c|c|c|c|}
\hline approach $\rightarrow$ & $4 \mathrm{~cm}$ & $2 \mathrm{~cm}$ & $1 \mathrm{~cm}$ & weighted mean & unit \\
\hline $\operatorname{mean}(x)$ & 0.107 & 0.105 & 0.149 & 0.066 & {$[\mathrm{~m}]$} \\
$\operatorname{mean}(y)$ & -0.045 & 0.060 & -0.04 & -0.05 & {$[\mathrm{~m}]$} \\
$\operatorname{std}(x)$ & 0.145 & 0.148 & 0.165 & 0.123 & {$[\mathrm{~m}]$} \\
$\operatorname{std}(y)$ & 0.081 & 0.088 & 0.087 & 0.076 & {$[\mathrm{~m}]$} \\
$\operatorname{mean}\left(\left|v_{x}\right|\right)$ & 0.146 & 0.095 & 0.084 & 0.075 & {$[\mathrm{~m} / \mathrm{s}]$} \\
$\operatorname{mean}\left(\left|v_{y}\right|\right)$ & 0.159 & 0.106 & 0.09 & 0.072 & {$[\mathrm{~m} / \mathrm{s}]$} \\
$\operatorname{std}\left(\left|v_{x}\right|\right)$ & 0.118 & 0.071 & 0.065 & 0.058 & {$[\mathrm{~m} / \mathrm{s}]$} \\
$\operatorname{std}\left(\left|v_{y}\right|\right)$ & 0.117 & 0.083 & 0.072 & 0.057 & {$[\mathrm{~m} / \mathrm{s}]$} \\
\hline
\end{tabular}

TABLE I

EFFECT OF MATCHING ALGORITHM ON POSE STABILITY OF THE ROBOT.

equal to run a Kalman filter having a large uncertainty on the prediction. Whereas including a more sophisticated model for the prediction would lead to better estimates, using this simplistic strategy was sufficient for our purposes.

\section{B. Localization and SLAM}

If a map of the environment is known a priori, pure localization (in contrast to SLAM) is sufficient for estimating the remaining $4 \mathrm{DOF}$ of the quadrotor. We estimate the $2 \mathrm{D}$ position $(x, y, \psi)$ of the robot in a given grid-map by MonteCarlo Localization [15]. The idea is to use a particle filter to track the position of the robot. Here, we sample the next generation of particles according given the relative movement estimated by the scan matcher and evaluate the current particle using likelihood fields [31].

Our system can acquire models of unknown environments during autonomous or manual flights by simultaneous localize and map the environment. The goal of a SLAM algorithm is to estimate both the vehicle position and the map of the environment by processing a sequence of measurements acquired while moving in the environment. Even when a map is known a-priori, a local map is needed until the robot is localized if the robot is running autonomously. In our system we use a popular graph-based SLAM algorithm. The idea of these types of algorithms is to construct a graph from the measurements of the vehicle. Each node in the graph represents a position of the vehicle in the environment and a measurement taken at that position. Measurements are connected by pairwise constraints encoding the spatial relations between nearby robot poses. These relations are determined by matching pairs of measurements acquired at nearby locations. Whenever the robot reenters a known region after traveling for long time in an unknown area, the errors accumulated along the trajectory become evident. These errors are modeled by constraints connecting parts of the environment that have been observed during distant time intervals and are known in the SLAM community as loop closures. To recover a consistent map we use a stochastic gradient descent optimization algorithm that finds the position of the nodes which maximizes the likelihood of the edges. The optimization approach is discussed in detail in [16], and an open source version is available on OpenSLAM [4].

Again, we restrict our estimation problem to $4 \mathrm{DOF}$, since the attitude provided by the IMU is sufficiently accurate for our mapping purposes. Furthermore, we assume that the vehicle flies over a piecewise constant surface and that the indoor environment is characterized by vertical structures, like walls, doors, and so on. Although trash bins, office tools on a table or the table itself are violating this assumption using a 2D map is still sufficient for accurate mapping and localization. This arises from the fact that clutter in general is only visible in a small portion of the current measurement, whereas mapping f.e. the desk improves localization since there is a clear difference in $x-y$ between a desk and a nearby wall. Thus we restrict our approach to estimate a $2 \mathrm{D}$ map and a $2 \mathrm{D}$ robot trajectory spanning over $3 \mathrm{DOF},(x, y, \psi)$, i.e., we map all objects if they had an infinite extend. The estimate of the trajectory is the projection of the 6DOF robot motion on the ground plane, along the $z$ axis. We estimate the altitude of the platform once the 2D position and the attitude are known, based on the procedure described in the next section.

\section{Altitude Estimation}

Estimating the altitude of the vehicle in an indoor environment means determining the global height wrt. a fixed reference frame. Since the vehicle can move over non-flat ground, we cannot directly use the the beams $\mathbf{h}$ deflected by the mirror. Our approach therefore concurrently estimates the altitude of the vehicle and the elevation of the ground under the robot. In our estimation process, we assume that the $(x, y, \psi)$ position of the robot in the environment is known from the SLAM module described above. We furthermore assume that the elevation of the surface under the robot is piecewise constant. We call each of these connected surface regions having constant altitude a "level". The extent of each level is represented as a set of cells in a 2D grid sharing the same altitude.

Since our system lacks global altitude sensors like barometers or GPS to determine the altitude of the vehicle, we track the altitude of the vehicle over the ground and map different elevations by using a two-staged system of Kalman filters. Algorithm 1 describes our approach in an abstract manner.

In the first stage, a Kalman filter is used to track the altitude $z$ and the vertical velocity $v_{z}$ of the vehicle by combining inertial measurements, altitude measurements and already mapped levels under the robot. In the second stage, a set of Kalman filters is used to estimate the elevation of the levels currently measured by the robot. To prevent drifts in the elevation estimate, we update the altitude of a level only when the robot measures the level for the first time or whenever the robot reenters it (i.e., enters or leaves that particular level).

In detail, the first Kalman filter estimates the height state $\mathbf{z}=\left(z, v_{z}\right)$ and the corresponding uncertainty $\Sigma_{z}$. First, we predict the current altitude and velocity $\left(\hat{\mathbf{z}}_{t}\right)$ given the previous estimate, $\mathbf{z}_{t-1}, \Sigma_{z_{t-1}}$, and the acceleration measured by the IMU (see line 4 of Algorithm 1).

The beams deflected by the mirror can measure more than one level simultaneously. For instance, when flying over a table it can happen that one fraction of the beams is fully reflected by the table, some beams are partially reflected by the table and partially by the floor, whereas the remaining beams are fully reflected by the floor. We therefore search in the local vicinity of the current multilevel-map for all levels which could have generated one of the measured altitudes $h \in \mathbf{h}_{t}$ (assuming the robot's altitude is $\hat{\mathbf{z}}_{t}$ ). This step is indicated in line 5. 
If we found at least one correspondence, we use them to calculate a virtual measurement to the ground (see line 7). We use the matched levels from the current map and the corresponding beams to calculate a single measurement. In other words, we calculate the measurement we would obtain if no obstacles were present underneath the robot and use this information for the measurement update of the Kalman filter as shown in line 8 .

However, when the robot explores the environment, it can happen that none of the current beams $h \in \mathbf{h}_{t}$ falls into a confidence region of a level in the current map, i.e., $\mathbf{E}=\emptyset$. In this case, we cannot create a virtual measurement and thus are unable to perform a measurement update of the filter. The prediction, therefore, is then the best estimate of the robot's altitude as described in line 10 .

Given the estimated altitude of the robot, we can now update the current multilevel map. Recall that the beams deflected by the mirror can measure more than one level simultaneously. We therefore cluster them into neighboring sets. Each of these sets is assumed to originate from a single level and it is parameterized by the mean and the covariance matrix calculated by the beams in the set. The outcome of this process is the set $\mathbf{L}$ consisting of the estimated levels as indicted in line 13.

We assume measurements not falling into a confidence region of existing levels in the local neighborhood to be generated by a new floor level. These new floor levels can be directly included into the map, as shown in line 14 in algorithm 1. For all measurements, falling into the confidence region of a level in the map, there exist two possibilities. Either this level has been already seen in the previous time-step, i.e., the robot is flying over the table and thus it has seen the corresponding level before, or it is currently entering or leaving this particular level. In the latter case, we can use the current altitude estimate in order to update the altitude of the level in the map (line 15). The elevation of each level is tracked by an individual Kalman filter.

Since we explicitly store objects in 2D with an extend in $x-y$ rather than individual levels per cell, we seek for those levels present in the neighborhood of the map, that are explained by one of the measurements currently obtained. If such a level is found and not present at the current location, we extend this level to the current cell, as shown in line 16.

Note that the robot observes only a limited portion of the underlying surface. Thus it may also happen that the robot "joins" the surfaces of different levels to form a new one. Figure 3 illustrates this situation. Initially two levels corresponding to a chair (Level 1) and a table (Level 2) are identified (a). The robot then left the table behind, makes a turn, and flies over a different area of the same table. Since Level 2 is not mapped in the neighborhood of the current pose, our system creates a new level (for the same table), noted as Level 3 in (b). Finally, the quadrotor continues to the originally covered area of the table which introduces an intersection of the current Level 3 and the previously generated Level 2. As a consequence, it joins Levels 2 and 3 (see (c) and (d)).

When two levels, $L_{j}^{\prime}$ and $L_{k}^{\prime}$, having altitudes $h_{j}$ and $h_{k}$ and covariances $\sigma_{j}^{2}$ and $\sigma_{k}^{2}$ are merged, the Gaussian estimate $\left\langle h, \sigma^{2}\right\rangle$ of the joint level has the following values:

$$
\left\langle h=\frac{\sigma_{k}^{2} h_{j}+\sigma_{j}^{2} h_{k}}{\sigma_{j}^{2}+\sigma_{k}^{2}}, \quad \sigma^{2}=\frac{\sigma_{j}^{2} \sigma_{k}^{2}}{\sigma_{j}^{2}+\sigma_{k}^{2}}\right\rangle .
$$

This step is indicated in line 17 of Algorithm 1.

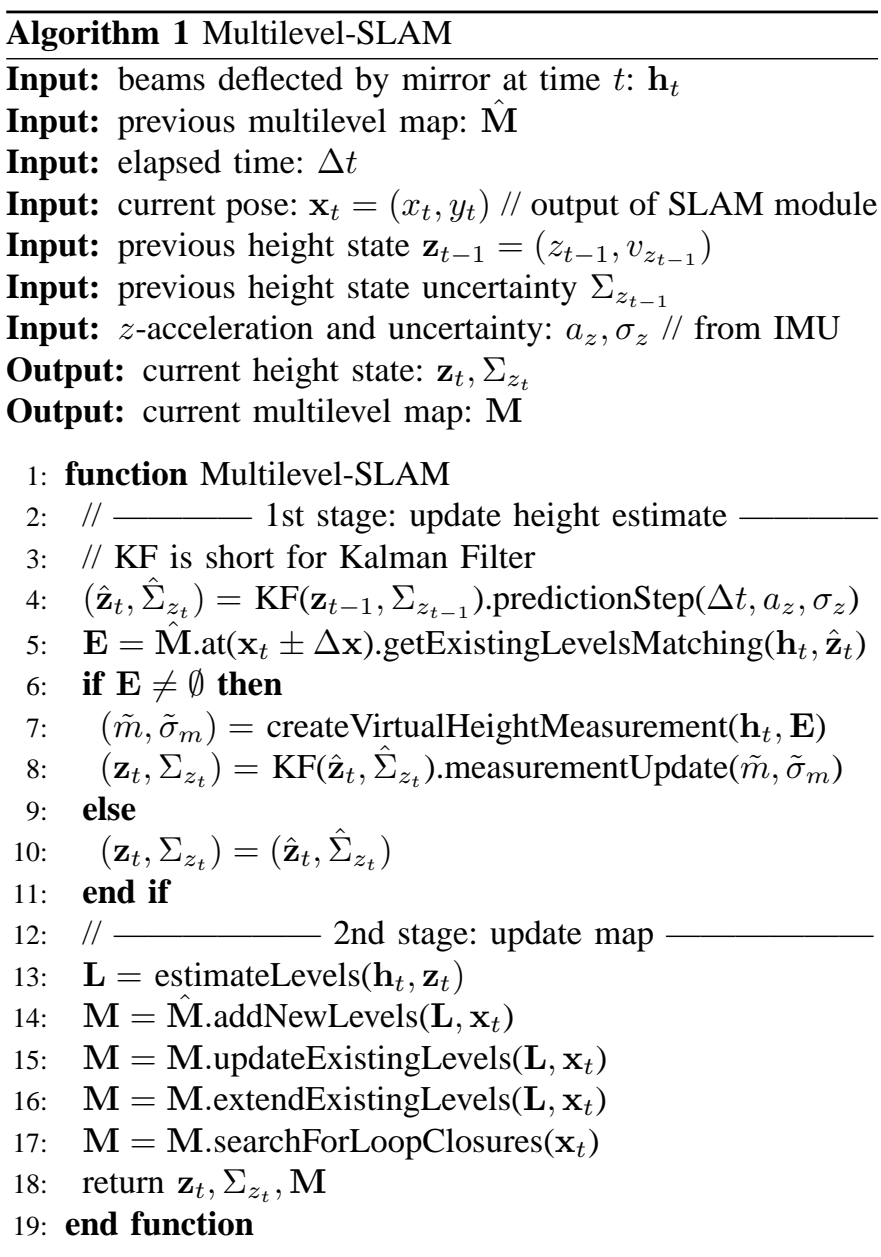

To summarize, we store a level as a set of 2D grid cells representing the area covered by the corresponding object. First, we estimate the current height of the robot given the known levels in the multi-level map. In a second step we update the map, given the estimated altitude of the robot. Here, a level is constantly re-estimated whenever the vehicle enters or leaves this specific level, and the data association is resolved by the known $(x, y, \psi)$ position of the vehicle. Finally, measurements not explained by any level present in the map are assumed to be generated by new levels which are then included in the map.

\section{High-Level Control for Pose and Altitude}

The high level control algorithm is used to keep the vehicle in the current position. The output of the control algorithm are variations in the roll, pitch, yaw, and thrust, denoted respectively as $u_{\phi}, u_{\theta}, u_{\psi}$ and $u_{z}$. The input are the position and the velocity estimates coming from incremental scanmatching. A variation of the roll translates in a motion along 


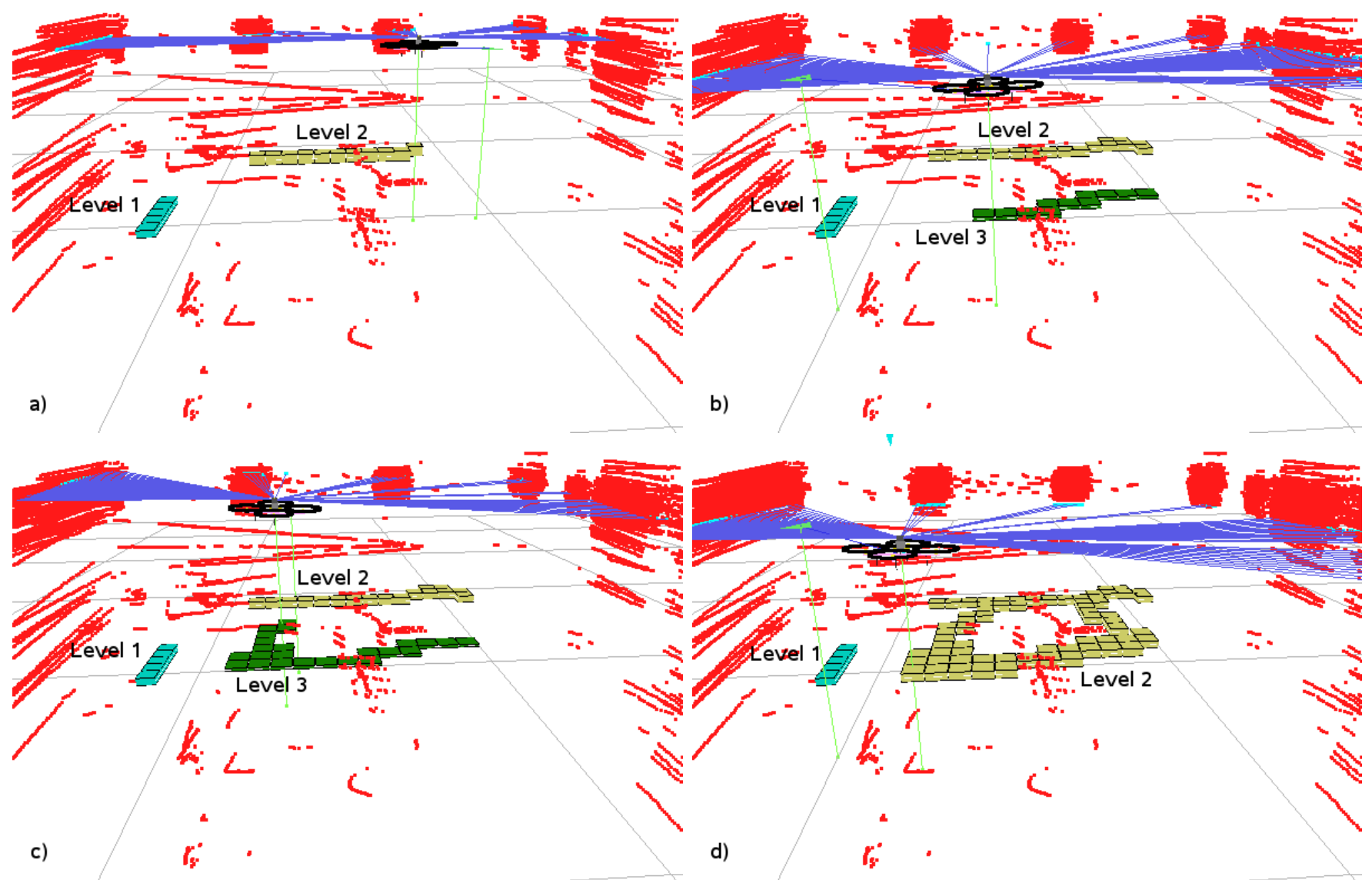

Fig. 3. Example of level joining during the estimation of the altitude of the vehicle and of the elevation of the underlying surfaces. Each level is represented as a set of contiguous cells in the $2 \mathrm{D}$ grid that share the same elevation. The robot starts exploring an office environment. Initially it recognizes two levels (Level 1, and Level 2), corresponding to a chair and a table (a). Subsequently it flies away from the table, turns back and flies over a different region of the same table (a). This results in the creation of the new Level 3. Then the robot keeps on hovering over the table until it approaches the extent of Level 2 which has the same elevation of Level 3, being originated by the same table. This situation is shown in (c). Finally the robot enters Level 2 from Level 3. Our system recognizes these two Levels to have the same elevation. Accordingly it merges them and updates the common elevation estimate (d).

the $y$ axis, a variation in the pitch results in a motion along the $x$ axis and a variation of the thrust results in a change in the vertical velocity. We separately control the individual variables via PID or PD controllers. Since in our case all control commands are dependent on the current pose estimate, our high level control module runs at a $10 \mathrm{~Hz}$, since the laser scanner provides measurements at exact this rate.

Note, that the Mikrokopter (and most of commercial available platforms) comes with low level controllers for roll, pitch, and yaw, thus we do not have to take care about the control of the individual motors, but of the control of commands resulting in a desired angle. In our particular case, the low level controller of the Mikrokopter quadrotor runs at $500 \mathrm{~Hz}$. Since commands for the yaw on common platforms result in how fast the quadrotor should turn and not how far, these parameters reflect the users wish of the robots aggressiveness wrt. the yaw rotation. In contrary to this, commands for roll and pitch result in a desired angle for which independent mapping functions must be learned. In order to learn the mapping for our quadrotor, we fixed one axis of the vehicle to an external frame allowing the vehicle to rotate along the other axis only. We learned the mapping function by monitoring the current angle measured by the IMU compared to the sent command. Our test bench for learning this mapping is shown
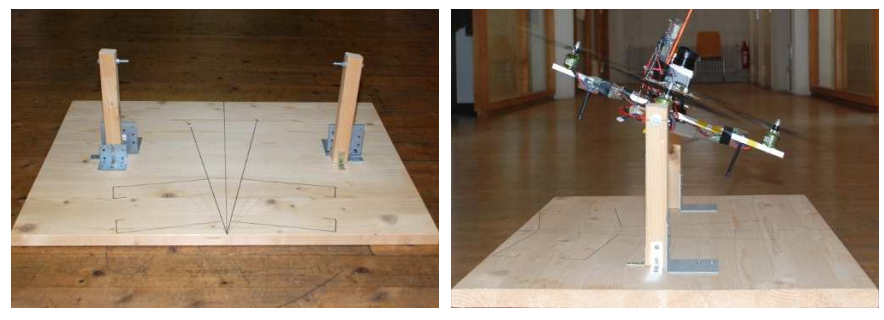

Fig. 4. Our test bench for learning a mapping between the command and the corresponding angle. This simple device allows for fixing one axis of the quadrotor and monitoring the other one using the IMU.

\section{in Figure 4.}

The calculated commands are sent directly to the microcontroller via RS232 which is in charge of the low level control (roll, pitch, and yaw) of the platform. For safety reasons, the user can always control the vehicle via a remote control and our system mixes the user and the program commands. During our experiments, we allow the programs to perturb the user commands by $\pm 20 \%$. In this way, if one of the control modules fails the user still has the possibility to safely land the vehicle without any loss of time since he does not need to press any button first.

In particular, we control the pitch and the roll by two independent PIDs that are fed with the $x$ and the $y$ coordinates 
of the robot pose. The control function in $x$ is the following:

$$
u_{\phi}=K_{p} \cdot\left(x-x^{*}\right)+K_{i} \cdot e_{x}+K_{d} \cdot v_{x},
$$

Here $x$ and $x^{*}$ are the measured and the desired $x$-positions, $v_{x}$ is the corresponding velocity, and $e_{x}$ denotes the error integrated over time. The control in the $y$ is analogous to the control in $x$. Note, that the integral part could be omitted (i.e., $K_{i}=0$ ), but we have encountered an improved hovering behavior if a small $K_{i}$ is used. This originates from the fact that in our case only integer values can be transmitted to the micro controller although the desired command is a float value.

We control the yaw by the following proportional controller:

$$
u_{\psi}=K_{p} \cdot\left(\psi-\psi^{*}\right) .
$$

Here $\psi$ and $\psi^{*}$ are the measured and desired yaw and $u_{\psi}$ is the control input.

The altitude is controlled by a PID controller which utilizes the current height estimate $z$, the velocity $v_{z}$, and the current battery voltage $U_{t}$ respectively. The control $u_{z}$ is defined as

$$
u_{z}=C\left(U_{t}\right)+K_{p} \cdot\left(z-z^{*}\right)+K_{i} \cdot e_{z}+K_{d} \cdot v_{z},
$$

with $K_{p}, K_{i}$ and $K_{d}$ being the constants for the P, I, and D part and $C\left(U_{t}\right)$ being the thrust command offset given the current battery voltage $U_{t}$ respectively. Here $z^{*}$ denotes the desired height and $e_{z}$ denotes the integrated error. Including a thrust command offset $C\left(U_{t}\right)$ allows us to treat the system as stationary, and therefore to use constant coefficients for the PID. We learned $C\left(U_{t}\right)$ by monitoring the thrust and the battery level of the vehicle in an expectation-maximization fashion. We started with a PID control without $C\left(U_{t}\right)$ and computed the average thrust command required to keep the current altitude using several test flights. For each battery level $U_{t}$ we computed the average thrust command required to keep the current altitude. In subsequent flights we used this offset as an initial guess for $C\left(U_{t}\right)$ and repeated the experiments resulting in an refinement for $C\left(U_{t}\right)$ until no major change in the estimated offset appeared.

\section{E. Path Planning and Obstacle Avoidance}

The goal of the path planning module is to compute a path from the current location to a user specified goal location which satisfies one or more optimality criteria and is safe enough to prevent collisions even in the case of small disturbances. Safety is usually enforced by choosing a path that is sufficiently distant from the obstacles in the map. Finally, due to the increased degrees of freedom of a flying vehicle compared to a ground robot, the path should be planned in 4DOF space instead of 3DOF. In our system we use $\mathrm{D}^{*}$ lite [22], a variant of the $A^{*}$ algorithm that can reuse previous solutions to correct an invalid plan rather than recomputing it from scratch. Since directly planning in 4DOF is too expensive for our system, we compute the path in two consecutive steps. First, we use $\mathrm{D}^{*}$ lite to compute a path in the $x-y-z$ space, but we only consider actions that move the robot in the 2D space $x-y$. For each $(x, y)$ location we know from the multi-level map the elevation of the surface underneath the robot. This known elevation is used to determine a possible change in altitude the robot would have to take when moving to a nearby cell. A change in altitude is reflected by increased traversability costs proportional to the distance in the $z$-direction. Furthermore, the cost function of a state $(x, y, z)$ of the robot depends on the distance of that location to the closest vertical obstacle in the map.

Once we have the $2.5 \mathrm{D}$ trajectory calculated with $\mathrm{D} *$ lite we augment it with the $\psi$ component. Since the laser scanner is heading forwards, it is desirable that the robot turns towards the direction of flight first to avoid collisions. On the other hand, we want the quadrotor to perform small maneuvers, like flying $10 \mathrm{~cm}$ backwards, without turning first. To achieve this behavior we calculate the desired angle which would result in flying forwards wrt. the local frame of the quadrotor. Trading off the costs of rotation versus costs of moving to the desired cell without rotating first allows the robot to perform pure sidewards or even backwards movements and thus prevents the vehicle from performing unnatural maneuvers.

Instead of switching to a new plan at every point in time, we try to re-use the existing solution whenever possible. A new plan is generated only when the actual plan is not valid anymore due to dynamic obstacles or when a certain period of time has been reached $(\Delta t=500 \mathrm{~ms})$. The latter constraint enables us to correct for detours in the trajectory that have been introduced to avoid obstacles that are no longer present. In our implementation, we use a grid resolution of $4 \mathrm{~cm}$. With these settings, the planner requires about 50-80 ms to compute a typical $10 \mathrm{~m}$ path from scratch. Re-planning can be done in less than $10 \mathrm{~ms}$. Dynamic obstacles are detected by the planner by considering the endpoints of the laser beams that are not explained by the known map (background subtraction). Additionally, we run a reactive obstacle avoidance module onboard in parallel based on potential fields [23].

\section{EXPERIMENTS}

In this section we present experiments that show the performances of each of the modules presented in the previous section, namely: localization, SLAM, multi-level mapping, autonomous pose stabilization, path planning, and obstacle avoidance. Videos of a series of different flights can be found on the Web [5].

\section{A. Localization}

Using 2D grid maps for localization enables our system to operate with maps acquired by different kinds of robots and not necessarily built by the flying vehicle itself. In this section we present an experiment in which we perform global localization of the flying quadrotor in a map acquired with a ground-based robot. This robot was equipped with a Sick LMS laser scanner. The height of the scanner was $80 \mathrm{~cm}$. Throughout this experiment, the UAV kept a height of $50 \mathrm{~cm} \pm 10 \mathrm{~cm}$ and the particle filter algorithm employed 5,000 particles. Given this number of particles, our current implementation requires $5 \mathrm{~ms}$ per iteration on a Dual-Core $2 \mathrm{GHz}$ laptop, while scan matching requires $5 \mathrm{~ms}$ on average. Figure 5 shows three snapshots of the localization process at three different points in time. The top image depicts the 


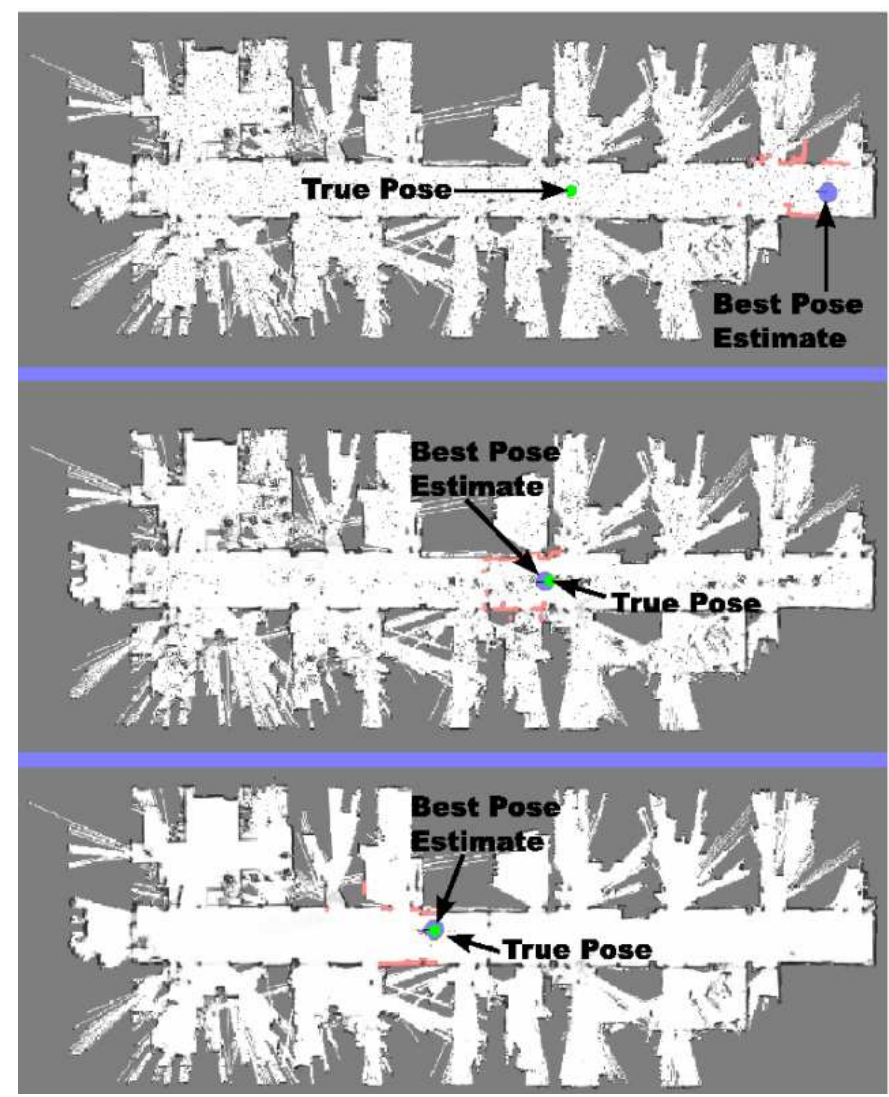

Fig. 5. Global localization of our quadrotor in a map previously acquired by a ground-based platform. The blue and the green circle highlight the current estimate of the particle filter and the true pose respectively. Particles are shown as black dots within the free space. Top: initial situation. Middle and bottom: after about $1 \mathrm{~m}$ and $5 \mathrm{~m}$ of flight. In the latter case, the quadrotor is localized.

initial situation, in which the particles were sampled uniformly over the free space. After approximately $1 \mathrm{~m}$ of flight (middle image), the particles start to focus around the true pose of the vehicle. After approximately $5 \mathrm{~m}$ of flight the quadrotor was globally localized (bottom image). The blue circle indicates the current estimate of the filter.

\section{B. SLAM}

We also evaluated the mapping system by letting the quadrotor fly four loops (approximately $41 \mathrm{~m}$ each) in a rectangular shaped building of approximate corridor size $10 \mathrm{~m} \times 12 \mathrm{~m}$. The result of our SLAM algorithm is shown in Figure 6. To quantitatively evaluate the accuracy of our mapping system we placed markers on the floor (labeled $1, \ldots, 4$ ) and manually landed the quadrotor close to the markers. Since we never perfectly landed on those we manually moved the quadrotor the remaining centimeters to match the predefined spots. This enables us to measure three types of errors: the re-localization error, the absolute positioning error and the error in openloop. The re-localization error is the difference between the current estimate and the estimate of the same real world pose in the previous loop. The error in open-loop is the relocalization error without enabling graph optimization. The absolute error is the difference between the estimated pose and the ground truth. To measure the absolute error we manually measured the relative locations of the markers and compared it to the positions estimated by the robot when landing to the

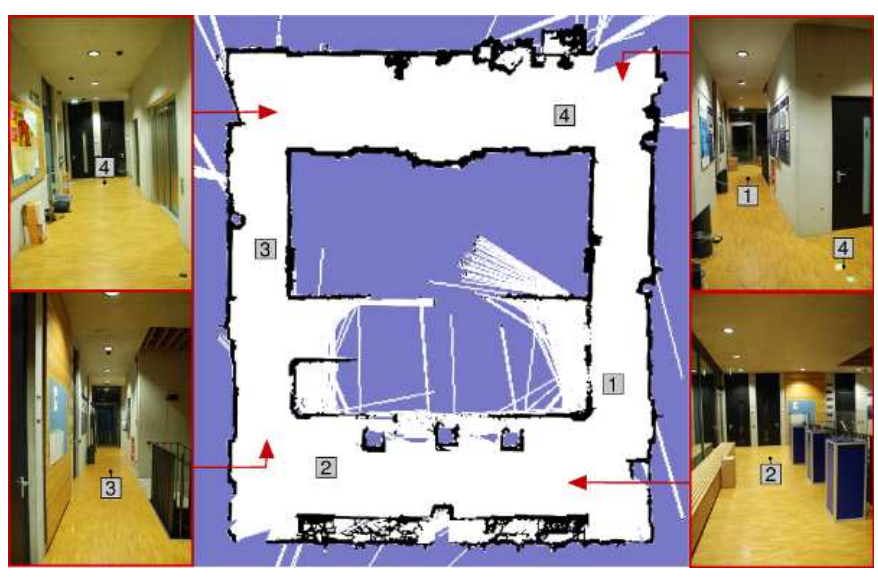

Fig. 6. Map of on office building built with our approach using the quadrotor. The labels 1-4 reflect the locations of individual markers used for evaluating the accuracy of our mapping approach. Red triangles indicate the pose of the corresponding camera images. The clutter in the bottom of the map originates from the seating containing horizontal slots (see bottom right image).

\begin{tabular}{|c||c|c|c|c|c|}
\hline marker & loop 1 & loop 2 & loop 3 & loop 4 & ground-truth \\
\hline$x_{1}$ & $1.11 \mathrm{~m}$ & $1.11 \mathrm{~m}$ & $1.11 \mathrm{~m}$ & $1.10 \mathrm{~m}$ & $1.11 \mathrm{~m}$ \\
$y_{1}$ & $-7.50 \mathrm{~m}$ & $-7.51 \mathrm{~m}$ & $-7.50 \mathrm{~m}$ & $-7.50 \mathrm{~m}$ & $-7.50 \mathrm{~m}$ \\
\hline$x_{2}$ & $-6.21 \mathrm{~m}$ & $-6.21 \mathrm{~m}$ & $-6.21 \mathrm{~m}$ & $-6.21 \mathrm{~m}$ & $-6.21 \mathrm{~m}$ \\
$y_{2}$ & $-9.21 \mathrm{~m}$ & $-9.21 \mathrm{~m}$ & $-9.21 \mathrm{~m}$ & $-9.21 \mathrm{~m}$ & $-9.21 \mathrm{~m}$ \\
\hline$x_{3}$ & $-7.85 \mathrm{~m}$ & $-7.85 \mathrm{~m}$ & $-7.85 \mathrm{~m}$ & $-7.85 \mathrm{~m}$ & $-7.85 \mathrm{~m}$ \\
$y_{3}$ & $-3.83 \mathrm{~m}$ & $-3.83 \mathrm{~m}$ & $-3.83 \mathrm{~m}$ & $-3.82 \mathrm{~m}$ & $-3.82 \mathrm{~m}$ \\
\hline$x_{4}$ & $-0.01 \mathrm{~m}$ & $-0.01 \mathrm{~m}$ & $-0.01 \mathrm{~m}$ & $-0.01 \mathrm{~m}$ & $0.00 \mathrm{~m}$ \\
$y_{4}$ & $-0.00 \mathrm{~m}$ & $-0.00 \mathrm{~m}$ & $-0.00 \mathrm{~m}$ & $-0.00 \mathrm{~m}$ & $0.00 \mathrm{~m}$ \\
\hline
\end{tabular}

TABLE II

ESTIMATED AND MANUALLY MEASURED LOCATIONS OF THE MARKERS FOR THE FLIGHT CONTAINING FOUR LOOPS IN TOTAL.

corresponding markers. Table II shows the manually measured and the estimated poses of the markers for all loops. As can be seen, both, the relative error between the individual loops and the global pose estimation wrt. the manually measured groundtruth have a maximum error of $1 \mathrm{~cm}$. In this experiment, the incremental mapping during the first loop was accurate enough $(<1 \mathrm{~cm}$ error) thus no optimization was needed since all subsequent loops were also re-localized in the existing map. We therefore also evaluated each loop independently of each other without enabling graph optimization. The results of the individual loop flights for marker 4 (origin) are shown in table III (first row). The worst flight (2nd loop) resulted in an error of approximately $0.37 \mathrm{~m}$ total distance to the origin. The remaining rows in table III show the effect of using different grid resolutions at the finest level of our hierarchical mapping approach on the accuracy of the individual loops.

\begin{tabular}{|c||r|r|r|r|c|}
\hline marker & \multicolumn{1}{|c|}{ loop 1 } & \multicolumn{1}{|c|}{ loop 2 } & \multicolumn{1}{c|}{ loop 3 } & \multicolumn{1}{c|}{ loop 4 } & finest resolution \\
\hline$x_{4}$ & $-0.01 \mathrm{~m}$ & $-0.35 \mathrm{~m}$ & $-0.08 \mathrm{~m}$ & $-0.17 \mathrm{~m}$ & \multirow{2}{*}{$0.01 \mathrm{~m}$} \\
$y_{4}$ & $-0.00 \mathrm{~m}$ & $0.12 \mathrm{~m}$ & $-0.07 \mathrm{~m}$ & $0.04 \mathrm{~m}$ & \\
\hline$x_{4}$ & $-0.42 \mathrm{~m}$ & $-0.59 \mathrm{~m}$ & $-0.36 \mathrm{~m}$ & $-0.64 \mathrm{~m}$ & \multirow{2}{*}{$0.02 \mathrm{~m}$} \\
$y_{4}$ & $0.20 \mathrm{~m}$ & $0.23 \mathrm{~m}$ & $0.11 \mathrm{~m}$ & $0.33 \mathrm{~m}$ & \\
\hline$x_{4}$ & $-0.91 \mathrm{~m}$ & $-0.59 \mathrm{~m}$ & $-0.54 \mathrm{~m}$ & $-0.60 \mathrm{~m}$ & \multirow{2}{*}{$0.04 \mathrm{~m}$} \\
$y_{4}$ & $0.28 \mathrm{~m}$ & $0.38 \mathrm{~m}$ & $0.29 \mathrm{~m}$ & $0.29 \mathrm{~m}$ & \\
\hline
\end{tabular}

TABLE III

COMPARISON OF SINGLE LOOPS FOR DIFFERENT GRID RESOLUTIONS.

\section{Multi-Level SLAM and Altitude Estimation}

In the following we show the typical behavior of our altitude estimation module. In this experiment, we let the robot fly 

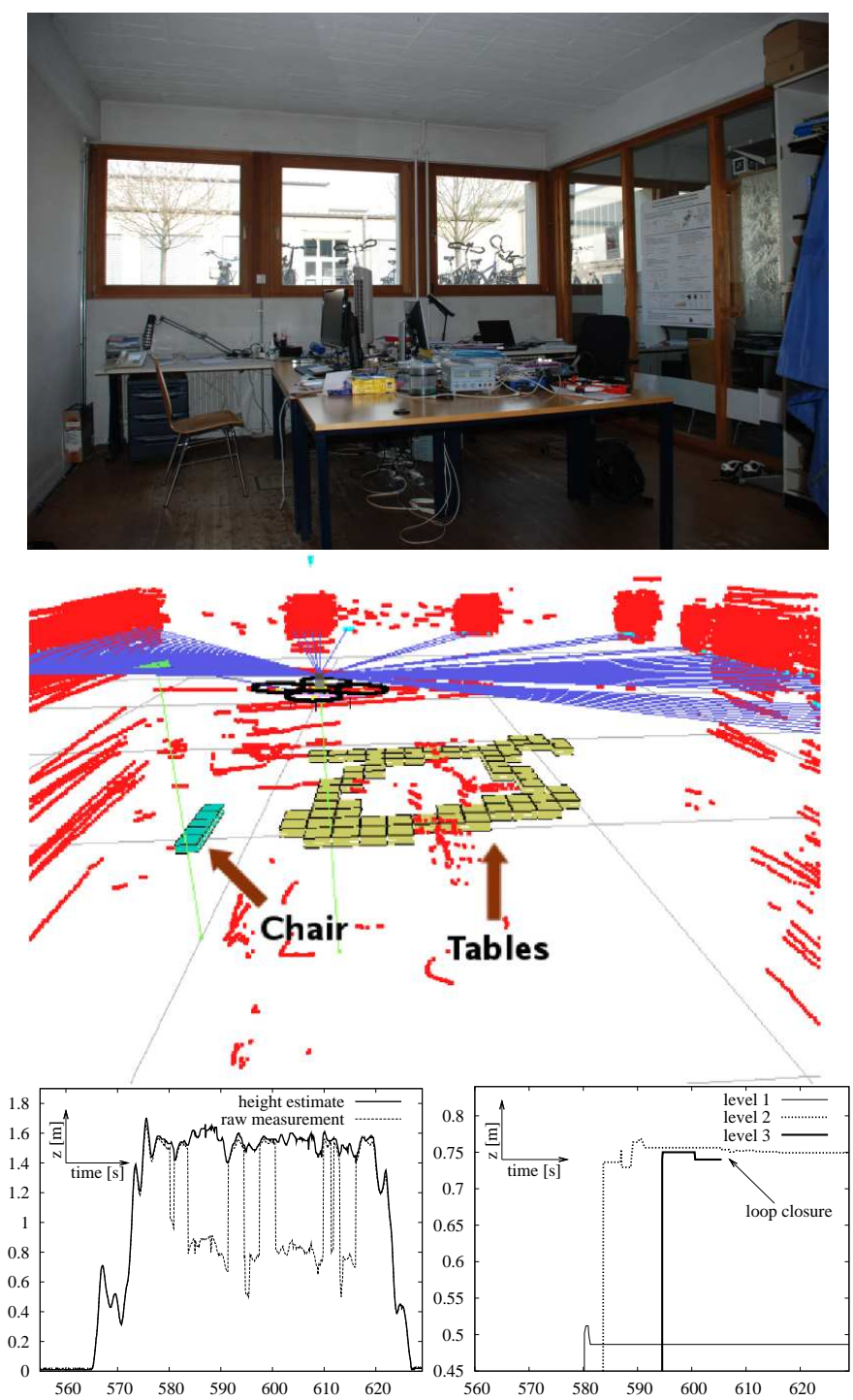

Fig. 7. Estimation of the global height of the vehicle and the underneath floor level. Whenever the quadrotor moves over a new level, the innovation is used to determine a level transition. The estimate of the height of each level is refined whenever the robot reenters that particular level. Top: the office environment. Middle: the corresponding map after autonomously flying over the vertical objects with a desired altitude of $150 \mathrm{~cm}$. Bottom left: a plot showing the estimated altitude of the vehicle over time versus the raw measurement. The corresponding estimated levels are depicted in the bottom right plot. Note, that Level 3 is merged with Level 2 after the loop closure.

autonomously in a typical office containing chairs, tables and lots of clutter. The chairs have a height of $48 \mathrm{~cm}$ and the tables are arranged next to each other having a height of $77 \mathrm{~cm}$. During this mission the system flew once over the chair and several times over the tables where it also flew in a loop. Figure 7 shows a snapshot of our multi-level mapping system during this mission. As can be seen from this figure, our algorithm correctly detected the objects at corresponding levels. The estimated heights of the chair and the tables were $48.6 \mathrm{~cm} \pm 2.7 \mathrm{~cm}$ and $74.9 \mathrm{~cm} \pm 2.8 \mathrm{~cm}$ respectively.

\section{Pose control}

Since the system is stabilized by independent controllers, we discuss the result of each individual controller.

a) Yaw control: For testing the yaw controller we set a desired yaw of $0^{\circ}$ and once in a while, we turned the helicopter

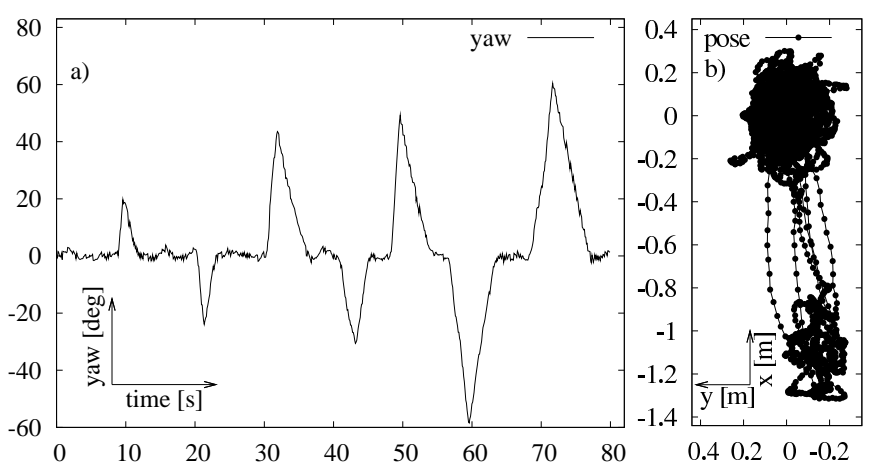

Fig. 8. Experiments for the autonomous stabilization of yaw (a) and pose (b). During the yaw stabilization experiment, the quadrotor was required to rotate to $0^{\circ}$, while the user manually turned the robot once in a while to a random orientation. Within the pose stability experiment the quadrotor was set to hover at $(0,0)$, but was manually moved backwards once in a while and required to fly autonomously back to the initial pose.

via the remote control. When the user released the remote control, the vehicle always returned back to its desired yaw with an error of $\pm 2^{\circ}$. Figure 8(a) plots the outcome of a typical run for yaw stabilization.

b) Altitude control: Similar to the experiment regarding the yaw, we ran an experiment to assess the behavior of the altitude control. In this test we set the desired altitude to $150 \mathrm{~cm}$. In the beginning the vehicle was hovering over the ground. After enabling the stabilization the vehicle started climbing to the desired altitude. The desired height was kept by the vehicle up to an error of $\pm 10 \mathrm{~cm}$. The results are shown in Figure 7. Note, that this experiment was performed while flying over different elevations.

c) $x, y$ control: Finally we show an experiment for the pose stabilization only. Note, that the pose stability is strongly affected by the latency of the system (i.e., the time needed to calculate the command given the laser data). Although incremental motion estimates take only $5 \mathrm{~ms}$ in average (with a maximum of $15 \mathrm{~ms}$ ) we have to deal with a latency of $120 \mathrm{~ms}$ in average due to the wireless transmission and due to the sensor buffer. A typical run including autonomous pose stabilization is shown in Figure 8(b). Here, the quadrotor was set to keep the initial pose of $(0,0)$ and once in a while, the user used the remote control to move the quadrotor around $1 \mathrm{~m}$ backwards. The quadrotor then autonomously moved back to the desired position. Depending on the latency in the system the pose oscillations are typically around $\pm 20 \mathrm{~cm}$.

\section{E. Path Planning and Obstacle Avoidance}

In this section we present an experiment demonstrating our algorithms for path planning and dynamic obstacle avoidance. The quadrotor was given a goal point approximately $5 \mathrm{~m}$ in front of it. A person was standing on the left (see the shaded area in Figure 9 entering the corridor when the quadrotor moved to its desired goal. The second image shows the situation when the person is completely blocking the robot's path. In this case the quadrotor hovered around the last valid way point since there was no valid plan to the goal anymore. When the person moved to the left again, the quadrotor was able to follow a de-tour as shown in the right image of Figure 9. Note, that the snapshots show the endpoints of the laser only. Although it looks like the quadrotor might have the 


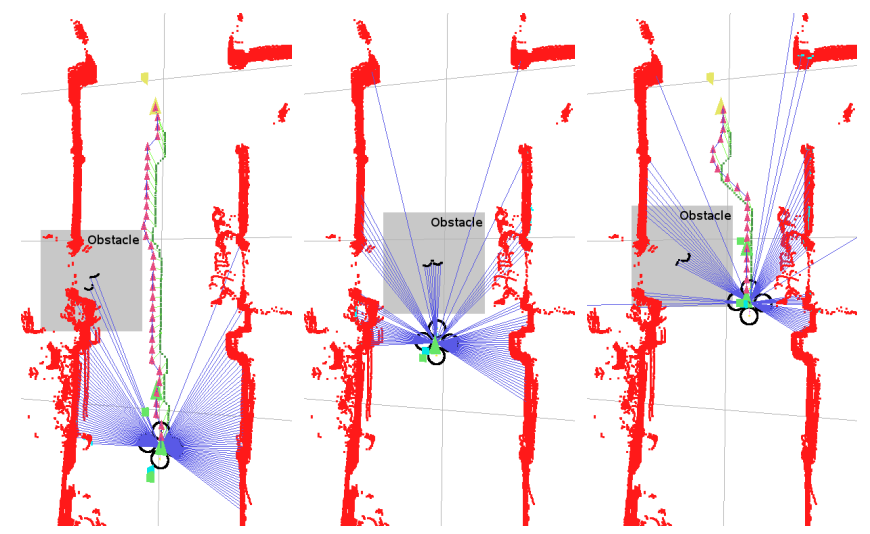

Fig. 9. Experiment for path planning and dynamic obstacle avoidance. The quadrotor is given a goal point $5 \mathrm{~m}$ in front of it. The planned path is shown in the left image. A person enters the corridor (shaded area) and blocks the robot's path, which results in an invalid plan. The quadrotor therefore hovers around the last valid way point (second image). In the third image the person moved back leaving the quadrotor enough space for a de-tour.

space to fly around the person in the second image, there is no valid plan due to the safety margins around the walls.

\section{CONCLUSION AND FUtURE WORK}

In this paper we presented a navigation system for autonomous indoor flying utilizing an open-hardware quadrotor platform. We described a complete navigation solution that approaches the different aspects of localization, mapping, pathplanning, height estimation, and control. Since we do not rely on special characteristics of the flying platform like the dynamics model, we believe that our system can easily be adapted to different flying vehicles. All modules in our system run on-line. However, due to the relatively high computational cost of some algorithms only a part of the software runs onboard on the ARM processor whereas the other part runs offboard on a laptop computer. Some preliminary tests make us confident that the whole system can run on-board using the next generation of embedded computers based on the Atom processor. We provided a wide range of experiments and some videos that highlight the effectiveness of our system. In future work we plan to add a time of flight camera into our system. We believe that this technology can be effectively integrated and will allow us to relax the assumption that the vehicle moves over a piecewise planar surface.

\section{ACKNOWLEDGEMENTS}

This work has been supported by the EC under contract number FP6-IST-034120 Micro/Nano based Systems

\section{REFERENCES}

[1] Carmen, http://carmen.sourceforge.net/.

[2] ROS - Robot Open Source, http://www.ros.org.

[3] Mikrokopter, http://www.mikrokopter.de/.

[4] OpenSLAM - Open Source Navigation Software Repository, http://www.openslam.org.

[5] http://ais.informatik.uni-freiburg.de/projects/quadrotor/.

[6] M. Achtelika, A. Bachrach, R. He, S. Prentice, and N. Roy. Autonomous navigation and exploration of a quadrotor helicopter in GPS-denied indoor environments. In Robotics: Science and Systems, 2008.

[7] S. Ahrens, D. Levine, G. Andrews, and J.P. How. Vision-based guidance and control of a hovering vehicle in unknown, GPS-denied environments. In Proceedings of the IEEE international conference on robotics and automation, pages 2643-2648, 2009.
[8] E. Altug, J.P. Ostrowski, and R. Mahony. Control of a quadrotor helicopter using visual feedback. In Proc. of the IEEE Int. Conf. on Robotics \& Automation (ICRA), 2002.

[9] A. Bachrach, R. He, and N. Roy. Autonomous Flight in Unknown Indoor Environments. Intl. Journal of Micro Air Vehicles, 1(4):217-228, 2009.

[10] S. Bouabdallah, C. Bermes, S. Grzonka, C. Gimkiewicz, A. Brenzikofer, R. Hahn, D. Schafroth, G. Grisetti, W. Burgard, and R. Siegwart. Towards palm-size autonomous helicopters. In International Conference and Exhibition on Unmanned Areal Vehicles (UAV), Dubai, UAE, 2010.

[11] S. Bouabdallah and R. Siegwart. Full Control of a Quadrotor. In Proc. of the IEEE/RSJ Int. Conf. on Intelligent Robots and Systems (IROS), 2007.

[12] O. Bourquardez, R. Mahony, N. Guenard, F. Chaumette, T. Hamel, and L. Eck. Image-based visual servo control of the translation kinematics of a quadrotor aerial vehicle. Robotics, IEEE TRO, 25(3):743-749, 2009.

[13] K. Celik, S.J. Chung, M. Clausman, and AK Somani. Monocular vision SLAM for indoor aerial vehicles. In Intelligent Robots and Systems (IROS), 2009., pages 1566-1573. IEEE, 2009.

[14] A. Coates, P. Abbeel, and A.Y. Ng. Learning for Control from Multiple Demonstrations. Proceedings of the International Conference on Machine Learning (ICML), 2008.

[15] F. Dellaert, D. Fox, W. Burgard, and S. Thrun. Monte carlo localization for mobile robots. In Proc. of the IEEE Int. Conf. on Robotics \& Automation (ICRA), Leuven, Belgium, 1998.

[16] G. Grisetti, C. Stachniss, and W. Burgard. Non-linear constraint network optimization for efficient map learning. IEEE Transactions on Intelligent Transportation Systems, 10:428-439, 2009. ISSN: 1524-9050.

[17] S. Grzonka, G. Grisetti, and W. Burgard. Towards a navigation system for autonomous indoor flying. In Proc. IEEE International Conference on Robotics and Automation (ICRA), Kobe, Japan, 2009.

[18] R. He, S. Prentice, and N. Roy. Planning in information space for a quadrotor helicopter in a GPS-denied environment. In Proc. of the IEEE Int. Conf. on Robotics \& Automation (ICRA), 2008.

[19] G. Hoffmann, DG Rajnarayan, SL Waslander, D. Dostal, JS Jang, and CJ Tomlin. The Stanford testbed of autonomous rotorcraft for multi agent control (STARMAC). The 23rd Digital Avionics Systems Conference (DASC)., 2, 2004.

[20] N.G. Johnson. Vision-assisted control of a hovering air vehicle in an indoor setting. Master thesis, Brigham Young University, 2008.

[21] C. Kemp. Visual control of a miniature quad-rotor helicopter. $P h D$, Churchill College University of Cambridge, 2005.

[22] S. Koenig and M. Likhachev. Fast replanning for navigation in unknown terrain. IEEE Transactions on Robotics, 21(3):354-363, 2005.

[23] J.-C. Latombe. Robot Motion Planning, pages 295-356. Kluwer Academic Publishers, 1991.

[24] Edwin Olson. Real-time correlative scan matching. In Proceedings of the IEEE International Conference on Robotics and Automation (ICRA), pages 4387-4393, Kobe, Japan, June 2009.

[25] P. Pounds, R. Mahony, and P. Corke. Modelling and Control of a QuadRotor Robot. Proceedings of the Australasian Conference on Robotics and Automation (ACRA), 2006.

[26] J.F. Roberts, T. Stirling, J.C. Zufferey, and D. Floreano. Quadrotor Using Minimal Sensing For Autonomous Indoor Flight. European Micro Air Vehicle Conference and Flight Competition (EMAV), 2007.

[27] N. Roy, M. Montemerlo, and S. Thrun. Perspectives on standardization in mobile robot programming. In Proc. of the IEEE/RSJ Int. Conf. on Intelligent Robots and Systems (IROS), Las Vegas, NV, USA, 2003.

[28] S. Scherer, S. Singh, L. Chamberlain, and M. Elgersma. Flying fast and low among obstacles: Methodology and experiments. The International Journal of Robotics Research, 27(5):549, 2008.

[29] A. Tayebi and S. McGilvray. Attitude stabilization of a VTOL quadrotor aircraft. IEEE TX on Control Systems Technology, 14(3):562-571, 2006.

[30] T. Templeton, DH Shim, C. Geyer, and SS Sastry. Autonomous visionbased landing and terrain mapping using an MPC-controlled unmanned rotorcraft. In 2007 IEEE International Conference on Robotics and Automation, pages 1349-1356, 2007.

[31] S. Thrun, W. Burgard, and D. Fox. Probabilistic Robotics, chapter Robot Perception, pages 171-172. MIT Press, 2005.

[32] S. Thrun, M. Diel, and D. Hahnel. Scan Alignment and 3-D Surface Modeling with a Helicopter Platform. Field and Service Robotics (STAR Springer Tracts in Advanced Robotics), 24:287-297, 2006.

[33] G.P. Tournier, M. Valenti, J.P. How, and E. Feron. Estimation and Control of a Quadrotor Vehicle Using Monocular Vision and Moire Patterns. AIAA Guidance, Navigation and Control Conference and Exhibit, pages 21-24, 2006. 


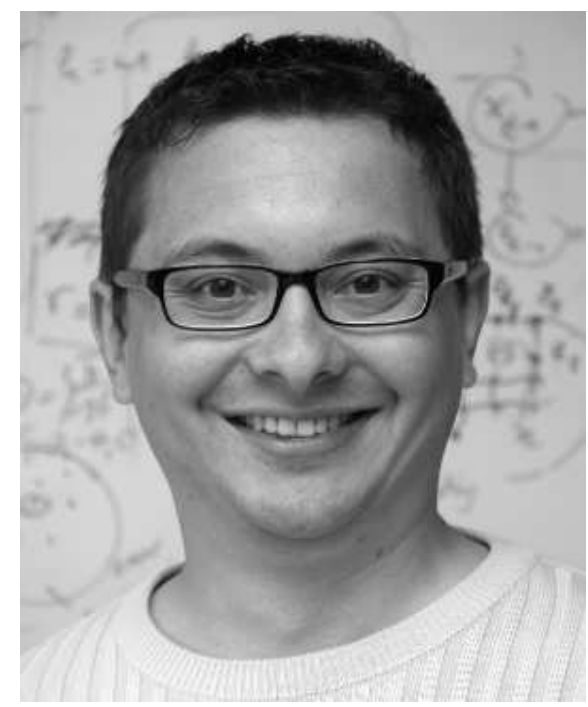

Slawomir Grzonka studied computer science at the University of Freiburg and received his Master degree in June 2006. He currently is a Ph.D. student in the Autonomous Intelligent Systems Lab of the University of Freiburg. His research interests lie in the areas of unmanned aerial vehicles, simultaneous localization and mapping, and human activity recognition. For his work on autonomous quadrotor systems he received best paper awards from the International Conference on Robotics and Automation (ICRA) in 2009 and from the International Conference and Exhibition on Unmanned Aerial Vehicles (UAV) in 2010.

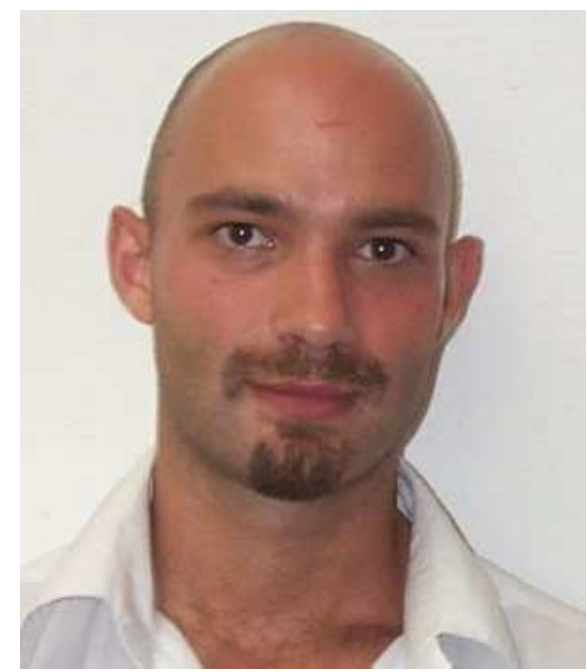

Giorgio Grisetti is assistant professor at the Dept. of Systems and Computer science of Sapienza University of Rome and member of the Autonomous Intelligent Systems Lab at the University of Freiburg. Before this, he was a Ph.D. student at University of Rome La Sapienza were he received his Ph.D. degree in April 2006. From 2006 to 2010, he was a postdoctoral researcher at the Autonomous Intelligent Systems $\mathrm{Lab}$ at the University of Freiburg. His research interests lie in the area of mobile robotic navigation, simultaneous localization and mapping, 3D mapping, and path planning.

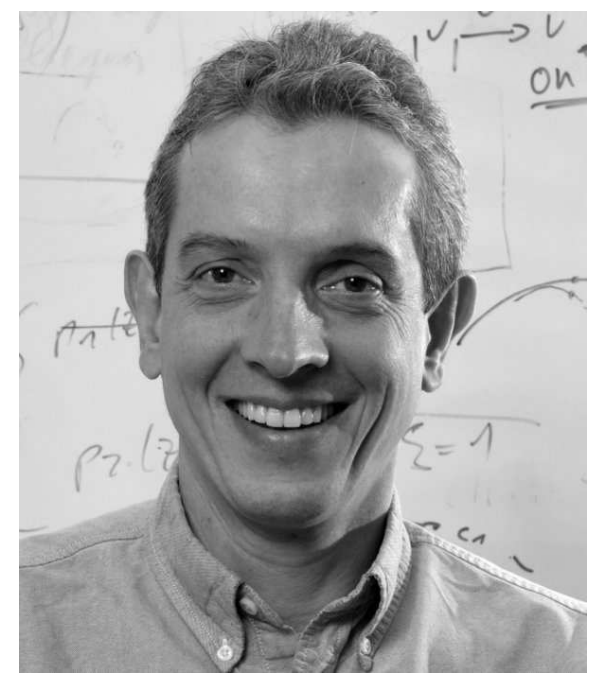

Wolfram Burgard is a full professor for computer science at the University of Freiburg, Germany where he heads the Laboratory for Autonomous Intelligent Systems. He received his $\mathrm{Ph} . \mathrm{D}$. degree in computer science from the University of Bonn in 1991. His areas of interest lie in artificial intelligence and mobile robots. In the past, Wolfram Burgard and his group developed several innovative probabilistic techniques for robot navigation and control. They cover different aspects such as localization, map-building, path-planning, and exploration. For his work, Wolfram Burgard received several best paper awards from outstanding national and international conferences. In 2009, Wolfram Burgard received the Gottfried Wilhelm Leibniz Prize, the most prestigious German research award. Recently, Wolfram Burgard received an Advanced Grant from the European Research Council. 